\title{
Novel optical frequency comb technology demonstrator for space
}

Tobias Lamour, F. Ye, O. Mandel, R. McCracken, J. Krauser, et al.

Tobias P. Lamour, F. Ye, O. Mandel, R. McCracken, J. Krauser, D. T. Reid, D. Weise, "Novel optical frequency comb technology demonstrator for space," Proc. SPIE 11852, International Conference on Space Optics - ICSO 2020, 1185239 (11 June 2021); doi: 10.1117/12.2599537

SPIE Event: International Conference on Space Optics - ICSO 2021, 2021, Online Only 


\section{International Conference on Space Optics-ICSO 2020}

Virtual Conference

30 March-2 April 2021

Edited by Bruno Cugny, Zoran Sodnik, and Nikos Karafolas
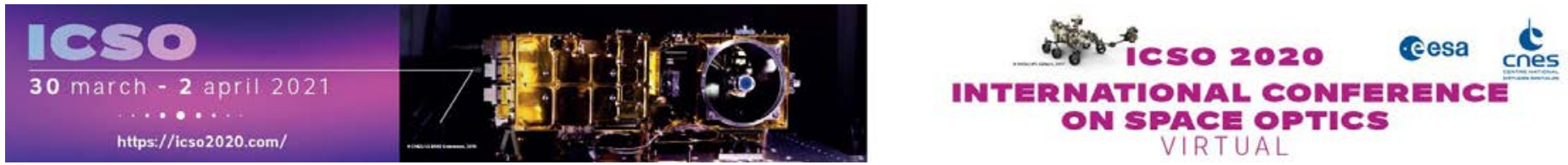

\section{Novel optical frequency comb technology demonstrator for space}

\section{Cesa isopousatang ecnes}




\title{
Novel Optical Frequency Comb Technology Demonstrator for Space
}

\author{
T. P. Lamour*a, F. Ye*b, O. Mandel ${ }^{\mathrm{a}}$, R. A. McCracken ${ }^{\mathrm{b}}$, J. Krauser $^{\mathrm{a}}$, D. T. Reid ${ }^{\mathrm{b}}$, D. Weise ${ }^{\mathrm{a}}$, \\ ${ }^{a}$ Airbus Defence and Space GmbH, Claude-Dornier-Strasse; 88090 Immenstaad, Germany \\ ${ }^{\mathrm{b}}$ School of Engineering and Physical Sciences, Heriot-Watt University, Edinburgh EH14 4AS, UK
}

\begin{abstract}
With this work, we present a new class of diode pumped solid-state based optical frequency comb (OFC) technology based on a Kerr-lens modelocked oscillator, which possesses robust self-starting capabilities. By combining this novel technology together with Airbus' capabilities of building highly stable, bonded optical space instruments, a preliminary optics module is demonstrated that produces sub-150-fs pulses with a repetition rate of $1.51 \mathrm{GHz}$, an output power of $12 \mathrm{~mW}$ and a central wavelength of $1078 \mathrm{~nm}$ with a bandwidth of $9.1 \mathrm{~nm}$. This novel technology is presented for the first time to provide some preliminary results and to show the capability of the technology. Operation has so far been demonstrated for tens of hours without performance degradation and it is expected to be significantly less sensitive against radiation than fibre based OFCs. All these features are packed in a mechanically robust and small micro-optical package, with the clear aim to deliver a compact solution fully suitable for space applications.
\end{abstract}

Keywords: Metrology, Frequency Comb, Frequency Reference, Microwave, Supercontinuum, Laser

\section{INTRODUCTION}

\subsection{Motivation}

The development of optical frequency combs (OFCs) for space applications has been underway for over a decade, in recent years focusing on fibre-based systems due to their great success in science and commercial applications [1,2]. However, demonstrations of space-borne fibre-based OFCs have been limited due to difficulties in realizing full stabilization with hands-free continuous operation under harsh environmental conditions over longer periods.

While the laser amplification process itself is quite robust to radiation, a major uncertainty that is far from being resolved is the radiation sensitivity of the nonlinear processes inside the fibre resonators. The impact on the delicate mode-locking processes is known to be serious, as both laser-gain and mode-locking processes take place along a distributed volume, where minute changes due to sensitivity to radiation or stress accumulate quickly, potentially rendering the mode-locking mechanisms in fibre-based system inoperative.

Solid-state systems on the other hand suffer from technical issues such as mechanical sensitivity that is overcome by the "semi-monolithic" nature of fibre-based systems. Much more severe though, are lifetime issues of their mode-locking media, usually based on (or at least supported by) a semiconductor saturable-absorber mirror (SESAM), known to have long-term stability issues with a constant need for manual interaction and replacement - a no-go for space operation.

A second branch of solid-state based OFCs employs Kerr-lens mode-locking (KLM). This nonlinear process happens inside a tiny fraction of a usually 1-4 mm sized laser gain crystal. Interestingly, this mechanism is known to deliver pulses with the lowest noise properties, ideally suitable for ultra-precise metrology applications. Only in some designs is the KLM process self-starting, and otherwise it needs some external support (i.e. a SESAM). This fact introduces a serious practicality issue - and minute changes in gain or pump power can lead to irrecoverable failure.

In this work, we introduce a new class of diode pumped solid-state-based OFC technology employing a Kerr-lens medium, whose design possesses robust self-starting capabilities that make it immediately attractive as a solution addressing the above-mentioned problems while occupying a significantly smaller footprint than a fibre oscillator.

*these authors contributed equally to the work. CONTACT: tobias.lamour@airbus.com 
We are convinced that this new OFC technology has the potential to serve as a system-enabling technology, which will allow time- and frequency-metrology applications of OFCs to be fully exploited into space applications.

\subsection{Novelty}

The present system integrates two disruptive approaches at once that make it especially attractive for space applications.

The laser oscillator employs an $\mathrm{Yb}: \mathrm{Y}_{2} \mathrm{O}_{3}$ (Yb:ceramic) gain medium, which provides access to the Kerr effect used for mode-locking inside the OFC cavity. This process is known to deliver the shortest optical pulses due to its high bandwidth and shows the lowest phase noise of all mode-locking mechanisms available to date. A further important benefit is that the process takes place in a tiny volume across the 2 -mm-long gain substrate, and is not distributed across the entire resonator, as is the case for fibre-based systems. This class of laser gain medium generally provides much better resilience to radiation due to their crystalline nature [3] than it is the case for fibre systems. This vulnerability has been a constant concern since the beginning of the fibre OFC concept, and is still an unsolved problem [4]. Therefore, this technology is expected to be much better suited for space-based applications due to its radiation hardness.

As mentioned in the introduction, it is common for KLM-based systems to suffer from problems that are not easily to overcome. They are all, directly or indirectly, caused by the narrow parameter window in which the KLM process is available. Therefore, it is quite common to use in addition a SESAM to provide a reliable self-starting mechanism while still get access to the excellent performance of the Kerr process. However, a solution using a SESAM is no option for long-term operation since the performance degrades continuously and requires some additional mechanism to move the SESAM surface across the spot. Reliable and robust self-starting capabilities and zero maintenance operation are both crucial assets for a successful space proven concept.

The presented system adopts a high-nonlinearity Yb-doped ceramic gain medium that, with appropriate cavity design, offers a significantly increased operation window in which KLM is self-starting. This offers reliable operation without any degrading components in the cavity and enables a technology, which becomes the first choice for high-precision, low-noise, zero-maintenance optical metrology platforms.

The second radical change compared to existing concepts is the way the optical physics package is integrated. In close cooperation, the bonding capabilities developed in the Laboratory of Enabling Technologies (LET) at Airbus Friedrichshafen were adjusted such that micro-optical components can now be placed and precisely bonded on a common breadboard. This allowed the manufacturing of a semi-monolithic optical instrument assembly that offers an optimal passive stability with the simplest possible setup.

Together with its high repetition rate of $1.51 \mathrm{GHz}$, which exceeds by a factor of 6-15 the typical value for fibre oscillators, this design allows a very compact system that further enhances the stability improvements and passively reduces the acoustic and thermal noise figures. The high repetition rate has a further metrology benefit, offering easier access to individual comb modes without any need for complex wavemeters or filter cavities - a major asset for its space applicability. This benefit is not directly accessible for fibre-based systems since they cannot be built much shorter without a significant performance penalty.

The unique combination of approaches detailed above has allowed us to realize a practical demonstrator, which is now described in greater detail.

\section{ENGINEERING \& DEVELOPMENT}

\subsection{Laboratory demonstrator}

2.2 The design of the laser oscillator follows the concept presented by Endo et al. [5, 6]. It is based on a bow-tie ring cavity, with two $\mathrm{r}=-20 \mathrm{~mm}$ focusing mirrors surrounding the nonlinear gain medium, which is a 2 -mm-thick $3 \%$ $\mathrm{Yb}$-doped glass ceramic introduced at Brewster angle $\left(62.5^{\circ}\right)$ for the resonant wavelength of $1078 \mathrm{~nm}$. A plane mirror mounted to a piezo transducer allows the cavity length to be adjusted to tune the comb-mode spacing. A second plane mirror coated with a Gires-Tournois interferometer (GTI) coating provides a $\sim 550-\mathrm{fs}^{2}$ group-delay dispersion compensation for the entire cavity and serves as the output-coupling mirror, with a transmission of $\sim 0.5 \%$ at $1078 \mathrm{~nm}$. Thanks to the compensation of the GTI mirror, the group-delay dispersion of the whole cavity 
is $\sim 300 \mathrm{fs}^{2}$. The laser is pumped at $976 \mathrm{~nm}$ using a polarization-maintaining fibre-pigtailed laser diode. In Fig. 1, a layout of the pump arrangement and the cavity components is presented.

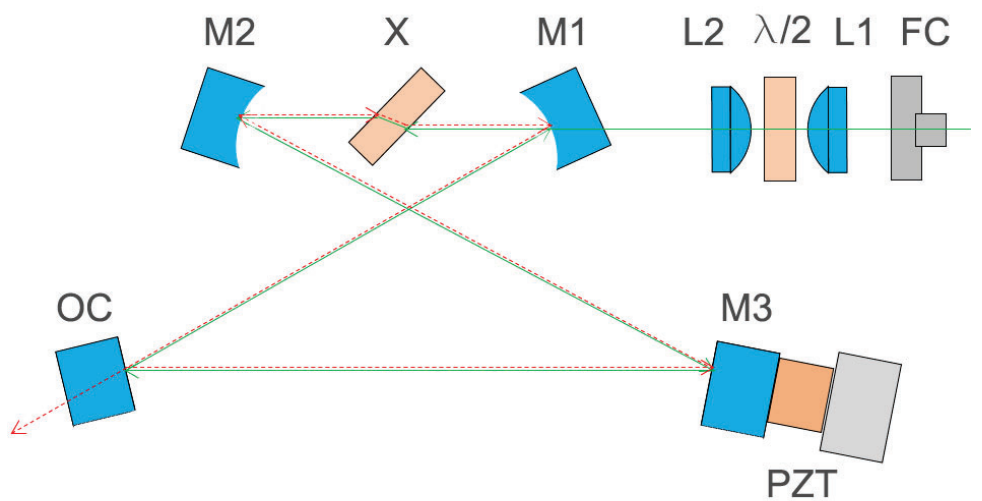

Figure 1. Cavity layout schematic with pump beam and cavity components is presented. The green solid line depicts the pump beam while the red dashed line shows the intra cavity beam as well as the out-coupled part of it. (FC: fibre coupler, L1/L2 collimation and focusing lens, $\lambda / 2$ : Half-wave-plate, X: Brewster-cut Yb-glass ceramic, M1: planeconcave pump mirror, M2: second plane-concave mirror, M3: plane mirror, OC: plain GTI and output coupler mirror, PZT: piezo transducer).

The cavity geometry determines the repetition rate of $1.51 \mathrm{GHz}$, corresponding to a cavity roundtrip path of $<20 \mathrm{~cm}$, and determines the frequency comb mode spacing once the resonator is in modelocked operation.

The first prototype was constructed using conventional laboratory opto-mechanics and some custom mounts for the crystal. Since the focusing section is very tight, special care is taken to manage the intra-cavity beam such that it is not clipped by the gain crystal due to the folding. In addition, compensation of the astigmatism introduced by the plane parallel Brewster plate of the gain crystal is achieved by the correct combination of mirror curvature and mirror tilt. This is optimised to generate a symmetrically round output beam close to diffraction limit.
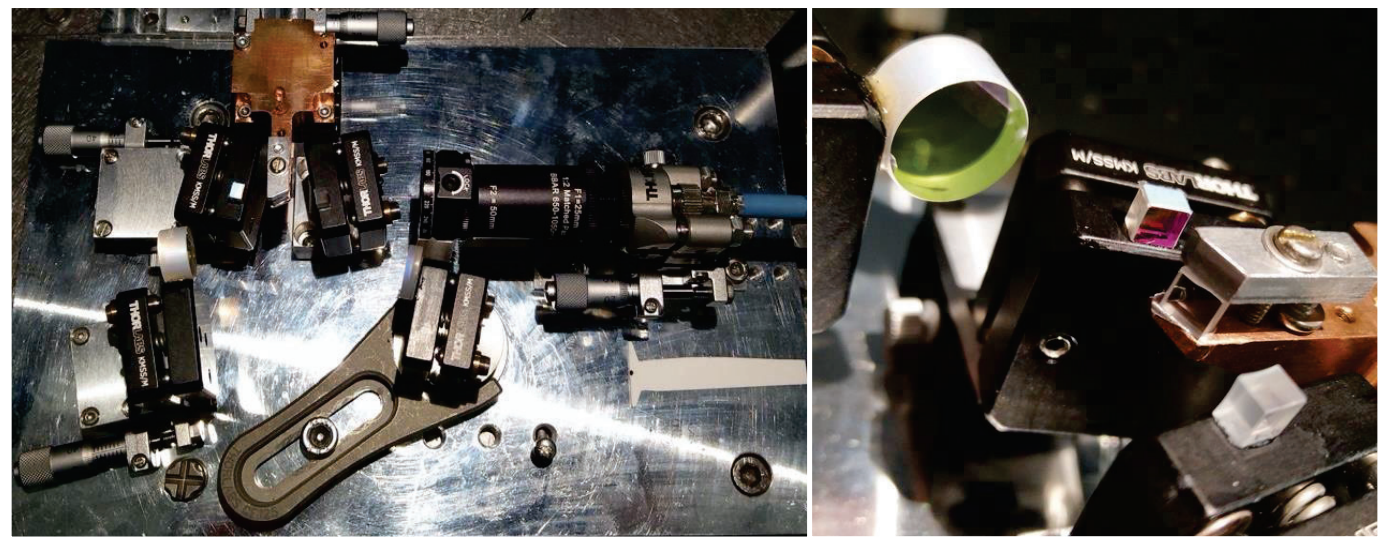

Figure 2. Left: An early lab set-up on a dedicated breadboard. Right: Close-up of the focusing section including the laser crystal.

The pump laser is a 980-nm telecom-qualified laser diode, which is terminated in FC/PC-connectorized polarizationmaintaining single-mode fibre. The laser diode produces a maximum output power of $900 \mathrm{~mW}$. Light from the FC/PC 
fibre connector is re-imaged through one of the curved cavity mirrors and into the Yb:ceramic gain medium by using a 2:1 telescope.

\subsection{Fusion of Airbus bonding expertise}

The optomechanical componentry represents a fundamental limitation of the system for space applications. The mirror mounts are subject to drift over time, introduce temperature sensitivity and are physically large. Nevertheless, the small dimensions of the 1.5-GHz laser mean that angular pointing changes in the mirrors have less of an impact than in larger systems, and we observed that even a system constructed entirely using conventional optomechanics remained in a selfstarting configuration over many months in a normal temperature-stabilized lab environment, needing no adjustment. This observation made it appealing to examine the performance of an entirely bonded laser resonator, however no offthe-shelf options were available to realise such a system.

The Airbus bonding expertise on ultra-stable optical instruments used for precision metrology was developed over many years in the Laboratory for Enabling Technologies (LET). This was a central ingredient for the successful preparation and development of instruments and missions such as the LISA Technology Package (Industrial Prime), the High Stability Laser Breadboard (Prime), the High-Precision Optical Metrology System, the Optical Metrology Laser Terminal, the Optical Bench Development for LISA (Prime), and the HSL for NGGM \& Laser Stabilization (System Verification) [7-14]. This heritage was applied to develop a means to precisely place and bond the components used in the OFC development.

\section{PROTOTYPE}

\subsection{First prototyping}

Following this concept, initial bonding tests were carried out allowing us to optimise a placement and alignment procedure. In addition, different adhesives and surface treatments were tested and evaluated. The final adhesive selected for the work achieved a balance between viscosity and strength, and had an unaccelerated curing time of 12-24h.

Some of the early test results are shown in Figure 3. For some of the in-situ tests, parts of the cavity were bonded and operated in order to prove the required stability during and after the curing process.
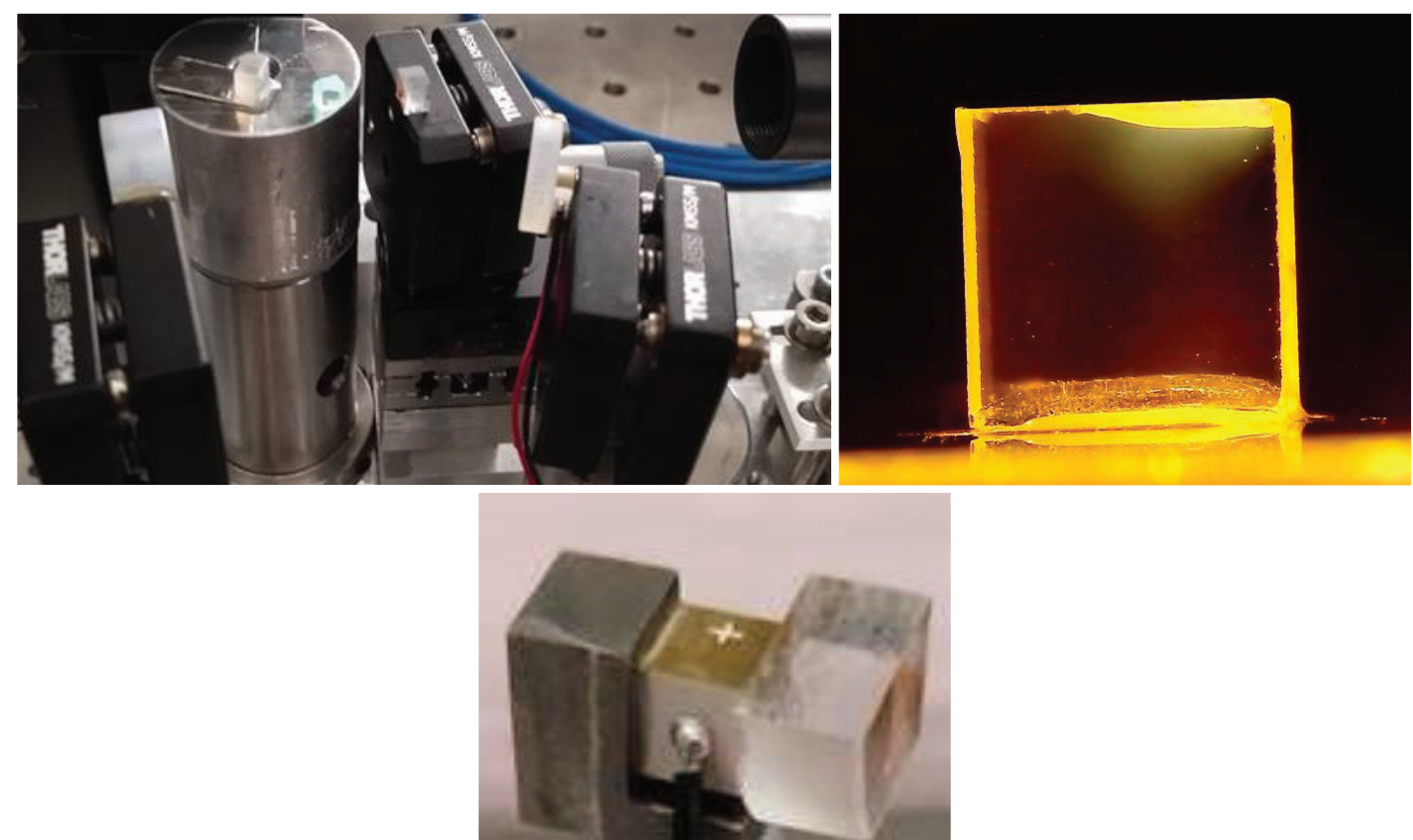

Figure 3. Clockwise, from top left: First bonding attempts to optimise the procedures and the application of the adhesive; further refinement achieving gain-crystal bonding; and bonding of the PZT-actuated mirror . 
Appling the adhesive to the small components needed several tests to optimise the portioning and correct application. Once this was solved, the bonded interfaces were routinely produced without major inhomogeneity or significant overor under-filling.

\subsection{Preliminary prototype breadboard}

The ultimate goal was to transfer a well-aligned laser oscillator systematically to a single optical breadboard. To achieve this, a specially designed jig was used to build and optimise the cavity in close proximity to the breadboard. Then the entire cavity with all components was bonded and cured in situ. Once this was achieved, the jig was removed, leaving the laser cavity perfectly mode-locking close to optimal performance. Figure 4 shows the resulting laser, with the $\mathrm{Yb}: \mathrm{Y}_{2} \mathrm{O}_{3}$ gain crystal and all four cavity mirrors bonded on the aluminium breadboard.

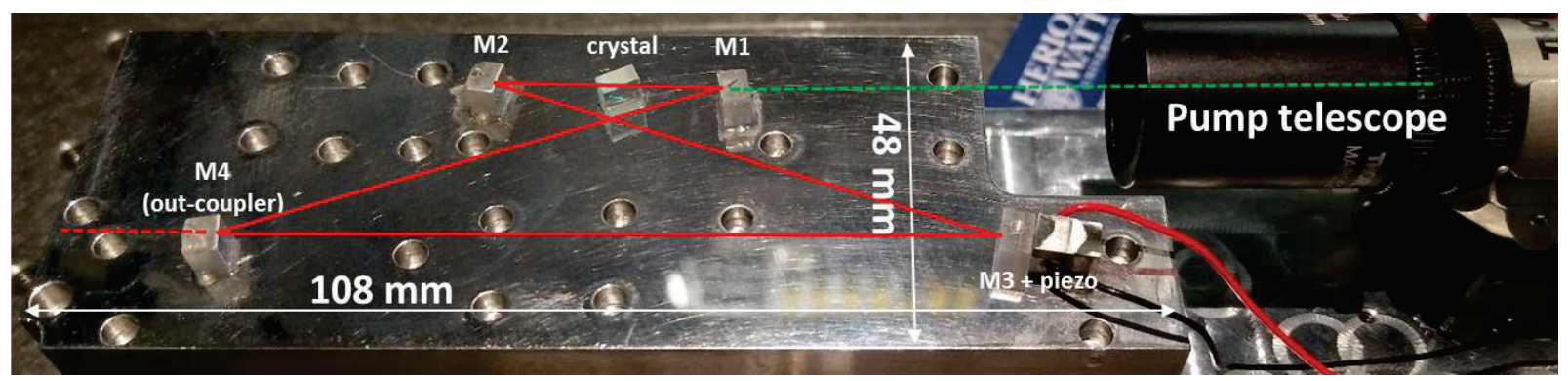

Figure 4. Final bonded and operational frequency comb cavity including the commercial pump telescope and the mirror-piezo transducer assembly for repetition rate locking. Dimensions of the breadboard are provided to give an impression on the footprint of the prototype system.

\section{CHARACTERISATION}

\subsection{Emission Spectrum}

Following the completion of bonding, the laser performance was characterised. The emission spectrum was centred at a wavelength of $1078 \mathrm{~nm}$ with a $-3-\mathrm{dB}$ bandwidth of $9.1 \mathrm{~nm}$. The laser modelocks in a soliton regime, evidence for which is the presence of Kelly sidebands, which are clearly visible in the spectrum, shown in Figure 5. The bandwidth limited pulse duration is assumed to $135 \mathrm{fs}$, corresponding to a $\operatorname{sech}^{2}(t)$ soliton at centre wavelength of $1078 \mathrm{~nm}$ and $9.1 \mathrm{~nm}$ bandwidth. This value is consistent with direct autocorrelation measurements, which have been performed on lasers of an identical design in our research group, although the particular laser here was not characterised by autocorrelation.

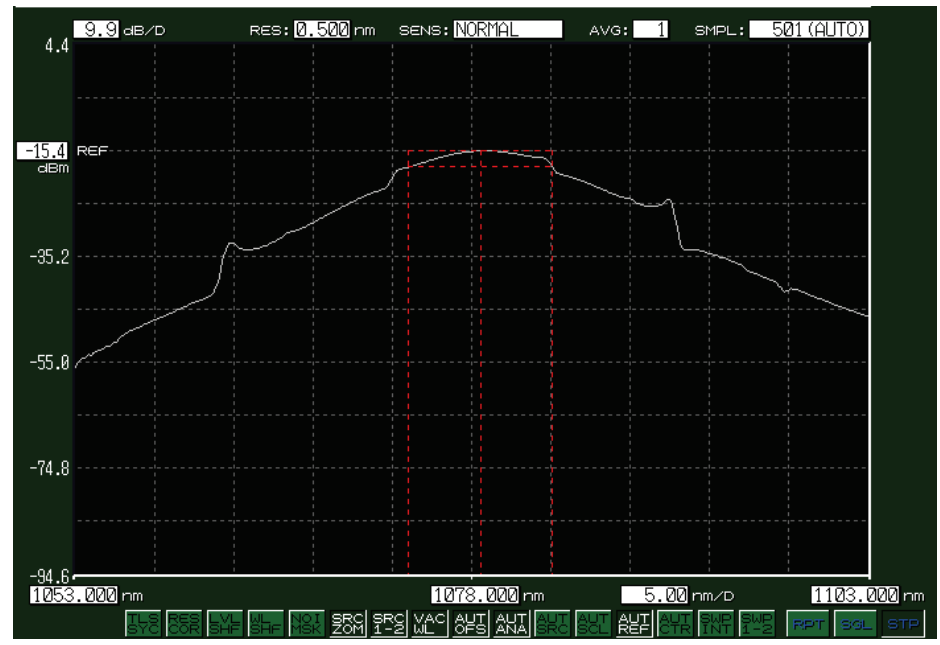

Figure 5. Spectrum of the operating laser, showing a centre wavelength of $1078 \mathrm{~nm}$ and a bandwidth of $9.1 \mathrm{~nm}$. 


\subsection{Output Power and Threshold}

Laser oscillation was obtained for pump currents above $100 \mathrm{~mA}$, and the laser provided stable modelocking for currents higher than $300 \mathrm{~mA}$. As Fig. 6(a) shows, the laser exhibited hysteretic behaviour for currents between 300-500 mA. In this regime, the ring oscillator operated in a unidirectional mode, transitioning into a higher gain configuration for pump currents approaching $500 \mathrm{~mA}$, for which a higher slope efficiency and output power was observed. At currents below this transition point, the laser emission bandwidth (Fig. 6(b)) increased at a rate of $0.024 \mathrm{~nm} / \mathrm{mA}$, reaching a plateau at the transition point, which we believe is when the pulse bandwidth became limited by the gain medium and / or the dispersion of the resonator. The transition may also correspond to a value of intra-crystal intensity that saturates the KLM process. We surmise that the transition is accompanied by a mode change inside the gain crystal leading to better overlap with the pump beam but reduced Kerr-lensing, allowing the pump current to be increased a little further before again saturating the KLM process. Up to this point, the pump current can then be reduced while the laser remains modelocked with higher output power. Increasing the pump current further causes the laser to enter a bidirectional modelocking state, in which the energy of the single unidirectional is divided into two counterpropagating pulses, of lower peak powers. In this regime, the KLM process appears to be unsaturated, the bandwidth of the pulses drop to lower values and the slope efficiency of each unidirectional output is reduced to that of the laser before the transition point.
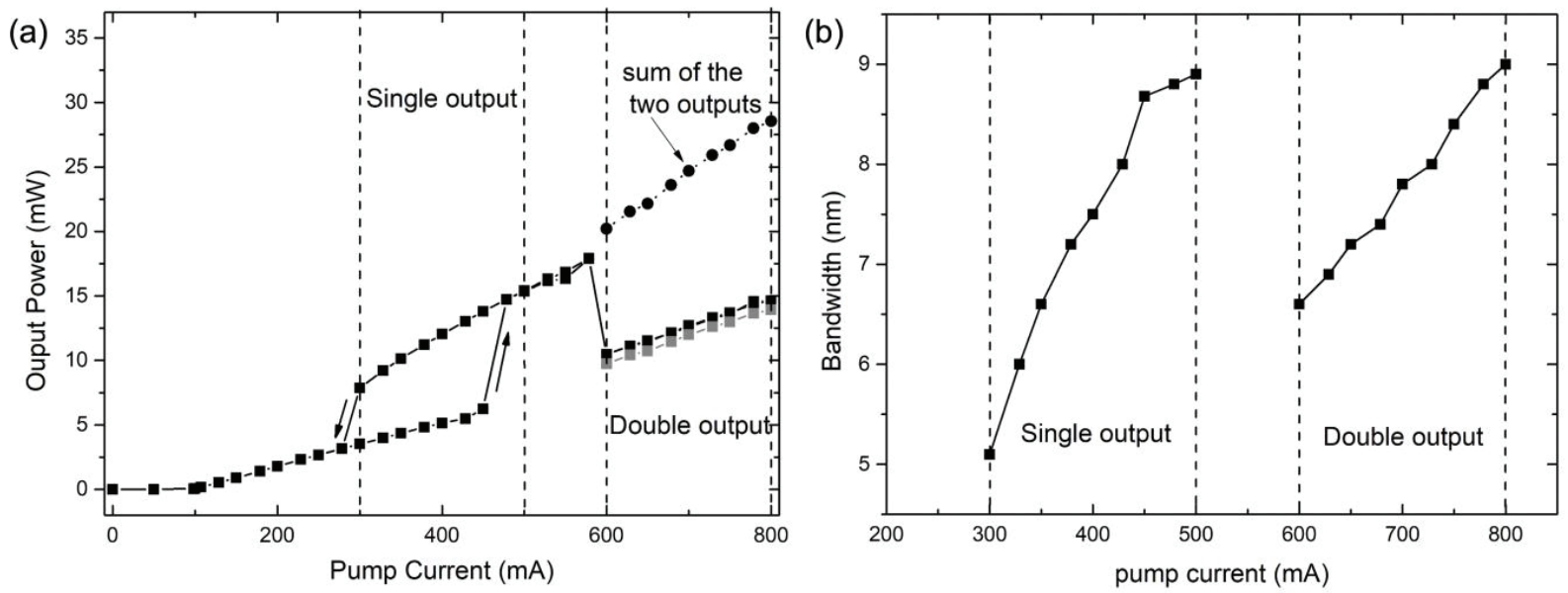

Figure 6. (a) Laser output power as the pump current was increased, and (b) corresponding behaviour of the laser emission bandwidth.

Stable operation was always possible for currents between $300-500 \mathrm{~mA}$, and it was in this range that all results were recorded.

\subsection{RIN}

The relative intensity noise (RIN) was compared between the pump laser and the Yb:ceramic OFC. Using a measurement system with a background rms noise of $0.03 \%$, the RIN of the pump laser from $1 \mathrm{~Hz}-1 \mathrm{MHz}$ was measured to be $0.13 \%$ (Fig. 7(a)), and the RIN of the femtosecond laser oscillator was measured to be $0.33 \%$ (Fig. 7(b)). The data show that the pump noise appears to couple into the RIN of the femtosecond laser, but because of the long upper state lifetime of $\mathrm{Yb}: \mathrm{Y}_{2} \mathrm{O}_{3}$, the sharp frequency contributions at $3 \mathrm{kHz}$ and $20 \mathrm{kHz}$ are dissipated.

The values obtained imply a true RIN of $0.3 \%$ for the laser, with $0.1 \%$ attributed to pump RIN. These figures compare very competitively with the best examples of other free-running femtosecond laser oscillators. Further reductions in laser intensity noise are expected to be possible by pre-compensating the pump RIN. 


\section{(a)}

(b)

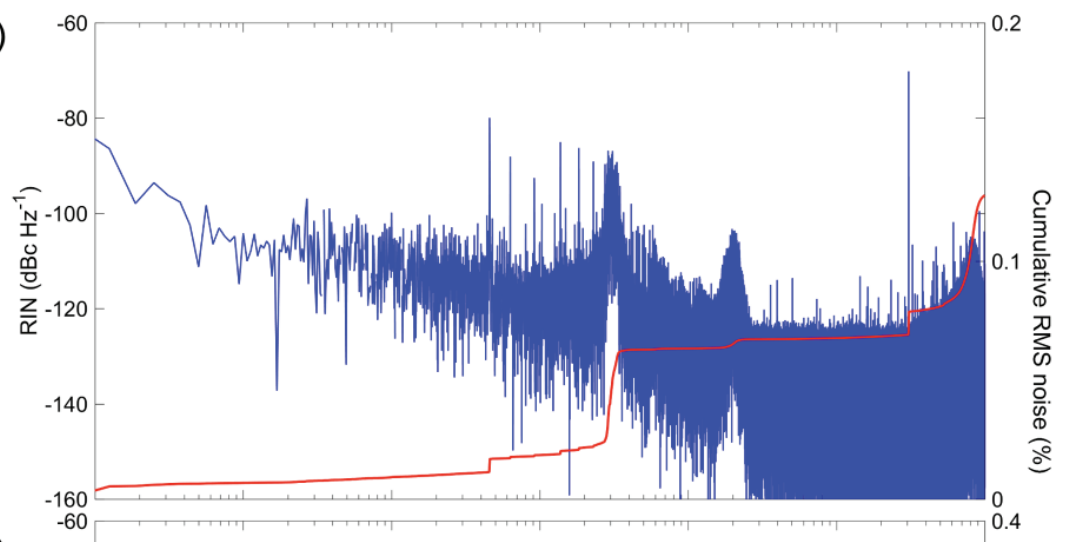

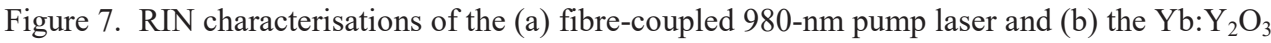
femtosecond laser. The blue trace is the power spectral density of the RIN and the red curve is the cumulative rms noise, showing what frequencies contribute most to the RIN.

\subsection{Repetition rate control}

The free-running laser has a repetition frequency $\left(f_{\text {rep }}\right)$ of $1.51 \mathrm{GHz}$ (Fig. $\left.8(\mathrm{a})\right)$ and the repetition frequency signal was sideband free with a SNR of $55 \mathrm{~dB}$. The piezo transducer bonded inside the laser cavity was characterised by applying $0-140 \mathrm{~V}$ and monitoring the resulting change in the laser repetition frequency. The observed tuning of the laser repetition frequency (Fig. 8(b)) was $15.7 \mathrm{kHz}$ and corresponds to a mirror displacement of $1 \mu \mathrm{m}$. This change corresponds to a shift of a comb line near $1078 \mathrm{~nm}$ by around $2.9 \mathrm{GHz}$ (almost $2 f_{\text {rep }}$ ) which would be sufficient to facilitate the stabilisation of a laser mode to any optical line reference. 

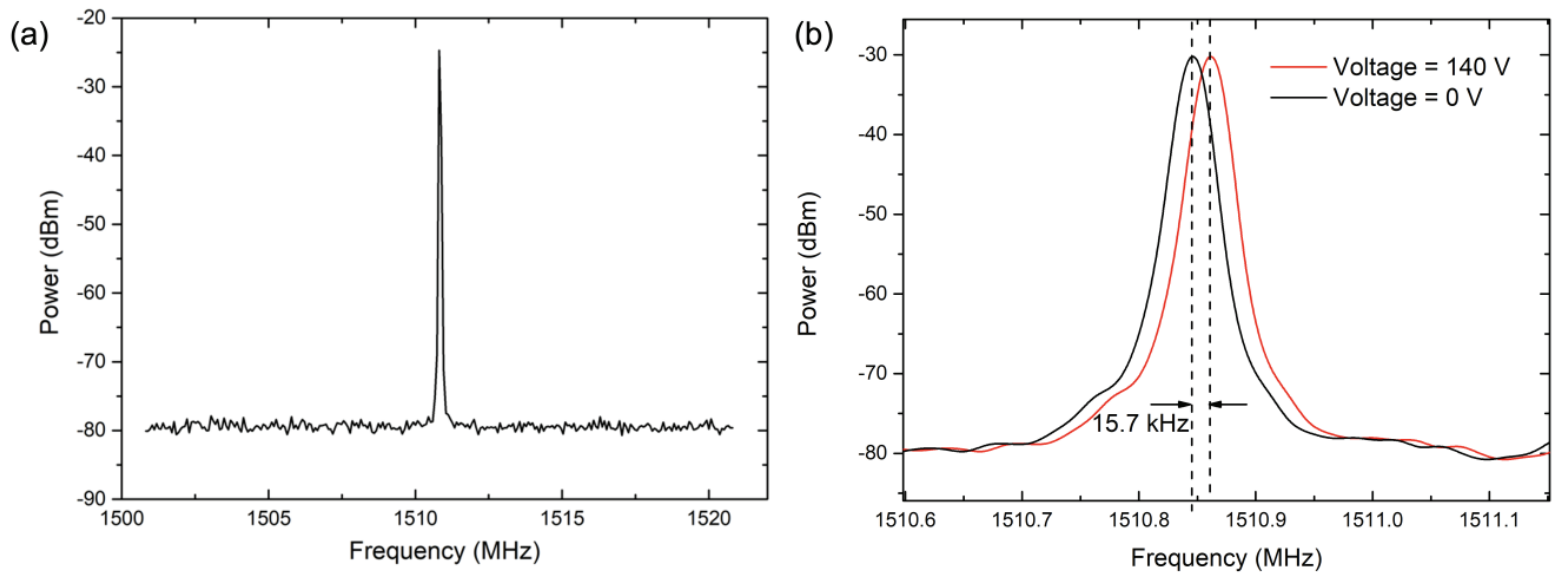

Figure 8. (a) Free-running power spectrum of the laser repetition frequency; (b) change of the repetition frequency measured on a RF spectrum analyser with zero and 140 volts applied to the PZT on which one of the cavity mirrors was mounted.

Full stabilisation of $f_{\text {rep }}$ was implemented by mixing the repetition frequency with the output from a microwave synthesiser at $1.51 \mathrm{GHz}$ and feeding back the difference-frequency signal to the PZT-mounted mirror.

\section{CONCLUSION AND OUTLOOK}

To our knowledge, this is the first example of a Kerr-lens-modelocked optical frequency comb based on semimonolithically bonding micro-optics to an aluminium breadboard. This directly-bonded configuration has the potential to provide the basis for an ultra-low-noise, high-stability, compact and extremely robust optical clock platform for space applications. Further work is now needed to implement the laser cavity and pump optics onto a single breadboard using the same bonding principles, resulting in a fibre-fed femtosecond laser package. Amplification is possible using polarization-maintaining core-pumped Yb-doped fibres, which in separate work we have demonstrated to increase the pulse energies to a level sufficient to achieve supercontinuum generation in SiN waveguides, a prerequisite to f-to- $2 \mathrm{f}$ interferometry and the stabilisation of the comb carrier-envelope offset frequency.

\section{References}

[1] M. Lezius et al., "FOKUS II - space flight of a vacuum compatible dual frequency comb system," CLEO Europe and European Quantum Electronics Conference, OSA Technical Digest (2019).

[2] M. Giunta et al., Optical Frequency Combs for Space Applications. STh4H.5. 10.1364/CLEO_SI.2016.STh4H.5 (2016).

[3] Buchs G, Kundermann S, Portuondo-Campa E, Lecomte S, "Radiation hard mode-locked laser suitable as a spaceborne frequency comb," Opt Express 23, 9890 (2015).

[4] ESA ITT ref. 9970: "Erbium-doped Radiation-hard Frequency Comb - EXPRO+" (awarded Feb.2020).

[5] Endo M, Ozawa A, Kobayashi Y, "6-GHz, Kerr-lens mode-locked $\mathrm{Yb}: \mathrm{Lu}_{-} 2 \mathrm{O} 33$ ceramic laser for combresolved broadband spectroscopy," Opt Lett 38, 4502. (2013).

[6] Endo M, Ito I, Kobayashi Y, "Direct 15-GHz mode-spacing optical frequency comb with a Kerr-lens modelocked $\mathrm{Yb}: \mathrm{Y}_{2} \mathrm{O}_{3}$ ceramic laser," Opt Express 23, 1276 (2015).

[7] O. Mandel et al., "Architecture and performance analysis of an optical metrology terminal for satellite-tosatellite laser ranging", Appl. Opt, 59, 653 (2020).

[8] H. Kögel et al., "Interferometric surface mapping of a spherical proof mass for ultra precise inertial reference sensors“, Appl. Opt. 55, 9830 (2016). 
[9] O. Mandel et al., Optical metrology terminal for satellite-to-satellite laser ranging. ICSO DOI $10.1117 / 12.2536072(2018)$.

[10] S. Ressel et al., "Ultrastable assembly and integration technology for ground- and space-based optical systems", Appl. Opt. 49, 4296 (2010).

[11] A. Schleicher et al., "In orbit performance of the LISA Pathfinder dag free and attitude control system", CEAS Space Journal, doi 10.1007/s12567-018-0204-x (2018).

[12] M. Chwalla et al., "Design and construction of an optical test bed for LISA imaging systems and tilt-to length coupling," Classical and Quantum Gravity 33, 245015-16 (2016).

[13] M. Chwalla et al., "Optical suppression of tilt-to-length coupling in the LISA long-arm interferometer", arXiv 2002.05669 (2020).

[14] M. Gohlke, et al., "Adhesive Bonding for Optical Metrology Systems in Space Applications,” 10th International LISA Symposium (LISAX) (2015). 